\title{
The Application of New Media in University Administration
}

\begin{abstract}
Shengkang He, Lu Liu
Nanchang Hangkong University, Jiangxi, Nanchang, 330036

3107000933@qq.com

739960103@qq.com

ABSTRACT

In recent years, with the rapid development of science and technology, the Internet and new media are also developing rapidly. Colleges and universities begin to use new media to assist their own administrative management. Compared with the traditional media, the new media has larger capacity, the carrier has been updated, the interaction is stronger, and the timeliness is faster. The traditional administrative work has some shortcomings that are hard to make up, but the emergence of new media just solves this problem. With the assistance of new media, the administrative workflow has become simplified, the efficiency of administrative management has been greatly improved, and the efficient administrative management system has been greatly improved, which is of great significance. This paper puts forward some suggestions on the administrative management of colleges and universities, hoping to help colleges and universities better carry out administrative management with the help of information technology.
\end{abstract}

Keywords: university administration, new media application

\section{新媒体在高校行政管理中的应用探索}

\author{
何声康, 刘璐
}

南昌航空大学 江西 南昌 330036

3107000933@qq.com

739960103@qq.com

\section{摘要}

最近几年, 科技进入高速发展阶段, 互联网和新媒体也随之快速发展着, 各高校开始运用新媒体来辅助自身 的行政管理。新媒体和传统媒体相比, 它具有更大的容量、载体进行了更新、互动性更强了、时效也更快

了。传统的行政工作有一些缺点难以弥补, 但是新媒体的出现正好解决了这一问题, 有了新媒体的辅助, 行 政工作流程变得简化, 行政管理的效率得到了大大的提高, 高效的行政管理体系得到了很大程度的完善, 这 是具有重大意义的。本文对高校的行政管理工作提出了一些建议, 希望能够借助信息化的手段, 帮助高校更 好地进行行政管理。

关键词: 高校行政管理; 新媒体应用

\section{1. 高校在进行行政管理过程中运用新媒体的 必要性}

新媒体是互联网时代衍生出来的一种新型的媒 介方式, 他正成为现在应用最为广泛的一项工具。新 媒体是一种媒介, 通过移动通信技术以及数字互联网 技术等向用户提供信息, 新媒体具有双向化、个性化
以及传播速度实时化的特点。目前新媒体发展速度很 快, 其覆盖的范围很广, 推广的成本低, 渠道多。新 媒体智能化, 它可以把行政事务信息化, 你凭借其特 点进行互动教学, 把传统的行政工作数据化、移动化、 便捷化以及系统化。这样一来, 可以减少办公成本, 还可以提升办公的效率。 


\section{2. 校行政管理过程中新媒体实际应用情况}

\section{1. 行政办公方面}

在文件传递方面, 以往传递文件是由专人按照一 定的工作流程进行传递，这样文件传递效率比较低， 文件内容时效性差。现在通过应用新媒体软件, 在线 上进行文件的传递, 即使相隔千里也只需几秒钟即可 实现上下传递，随时随地文件的签约者都可以阅读， 以及签批文件，不受时间和空间的限制，这样一来， 文件的处理效率得到大大的提高, 文件的时效性较强。 对于新闻行业来说, 新闻的时效性非常重要, 有了新 媒体这一媒介, 新闻的传播更加高效, 甚至还能通过 监控系统来控制风险, 防患于未然。还可以通过微信 公众号的一些服务模块, 进行饭卡充值, 水电缴费以 及学生考勤 ${ }^{[2]}$ 。

\section{2. 教学教务方面}

目前教育发展的主流有 “微教育” 以及慕课等, 凭借着新媒体的优势, 深受教师以及学生的喜爱。这 种网上课堂比线下课堂时长短, 只有线下课堂的一半, 有的甚至只有五分钟, 这些教学视频以新媒体为载体, 供学生任意使用, 学生可以随时随地, 所遇的进行学 习。老师, 把大量的教学视频放到网上, 学生可以按 照自己的兴趣爱好进行选择, 也可以采取直播的形式, 增加学生与老师之间的互动, 在网上平台上还可以发 布一些测试, 检验学生的学习成果 ${ }^{[3]}$ 。录播的课程学 生可以事先下载好, 即使没有网络, 也可以观看, 学 习不受时间空间的限制, 而且与传统教学方式相比, 非常新颖, 教学的创意感得到增强。

\section{3 在学生管理方面的运用}

高校在实行行政管理的过程中, 姜新媒体运用 到学生管理当中, 主要是通过企业微信号等方式。 通过企业微信号可以记录学生的日常考勤以及相关 的奖学金、助学金等, 学校也可以通过企业微信号 进行学生档案以及学生成绩记录等方面的管理。这 种通过新媒体的手段, 记录学生的日常生活和相关 的活动可以极大地降低记录成本和管理成本。

\section{3. 高校在行政管理过程中应用新媒体时所出 现的问题}

\section{1. 缺乏规范的建设标准, 改进的进度缓慢}

在高校进行建设数字化校园的初期, 其存在的主 要问题就是整体性不足, 院校自主研发的信息系统与 第三方软件系统没有办法协调合作, 这就需要不同的 平台来承载, 导致多个平台并存, 这样一来, 院校管 理起来比较复杂困难。在建设初期, 没有整体性, 后 期就需要花费更多的时间和精力来进行改进。而且各 院校指导建设的领导人员自身的数字化专业技能不
高, 难以科学合理地进行指挥 ${ }^{[5]}$ 。学校对人事的管理, 主要侧重于教师的管理, 技术人员的管理规定较少, 教师有职称竞争，但是技术人员没有这一竞争压力, 因此，高校信息技术研发团队发展缓慢。

\section{2. 在新媒体使用中的安全隐患}

\section{2. 1. 大数据信息安全隐患}

现如今, 大数据已经遍及整个社会, 信息化时代 的普及在校园中也同样重要。校园中几乎所有的信息 都在数据中心，因此，在共享信息的同时，对于信息 系统的防御和监控的保护也极为重要。信息化校园在 教学以及资料查询和管理方面提供了极大的便利。但 往往这些便利后面会存在一些安全隐患和漏洞, 像校 园网的普及, 为学生们提供了上网的便利, 但同时也 让同学们的信息有被泄漏的可能。微信企业号的广泛 利用，方便了同学们的信息流通，但也有人利用非法 手段从中获取用户个人信息, 导致数据丢失, 个人信 息受到威胁。总的来说，我们在享受信息化校园带来 便利的同时，更应该注重其背后的隐患。像各种基础 设备, 服务软件, 硬件设备等无故障碍, 都是数据中 心可能面临的风险。

\subsection{2. 网络攻击安全隐患}

网络安全问题不仅在商业圈中经常出现, 校园网 络安全问题也出现在大众的视野。虽然校园网络中并 不涉及国家机密或者商业机密等内容, 但是, 我国高 校是人才培养的集中区域, 其中所涉及的课题, 实验 项目等其中的信息价值也极为重要。因此, 校园网络 的攻陷, 也是一件极为严峻的问题。

\section{2.3 学校宽带资源不太稳定}

校园网络主要依赖于当地的运营商, 运营商的网 络运输速度决定了整个校园网络的稳定程度和运输 速度。而对于学校来说, 人口较多, 使用网络的人也 相对较多, 使用网络的时间也相对较多。因此, 拥有 一个相对靠谱和稳定的运营商对于整个校园网络的 正常运行是十分重要的。但是, 就目前的调研结果来 看, 大部分学校的网络是不太稳定的, 造成这种不太 稳定的结果是由于大多数学校属于单一运营商垄断, 这一点需要改进。最重要的是由于技术的不断增强, 而 WiFi 的特性导致了许多盗用身份以及攻击防火墙 等现象的出现, 严重的影响了同学们在进行新媒体使 用过程中的安全性。

\section{3 技术水平相对较低}

高校的信息化建设过程中, 大多数是依赖于软件 的运营商, 而没有自己的信息化建设系统, 这样子会 造成信息化的技术水平较低。而市场上的软件运营商 种类颇多，如果学校没有一定的规划或者没有一定的 前瞻性, 由于技术水平较低就会导致购买的失误。这 
种失误会给学校带来额外的金钱损失。如此之外, 由 于信息技术的水平参差不齐, 许多老师没有办法适应 这项新的技术，从而影响到整个教学的效果。这些由 于技术水平造成的损失，都是叒待解决的问题。

\section{4. 针对新媒体使用的应对策略}

\section{1. 建立完善的管理机制和法律法规}

有法可依, 违法必究, 不仅现代社会需要法律的 维护，虚拟网络社会也应该有法律的维持。因此，各 高校可以发布相关校园网络安全建设的法律法规, 帮 助新媒体建设和运行。完善相关管理制度, 合理利用 网络信息平台, 加强各部门对信息平台的维护。在新 媒体运行时, 严格遵循, 本校发布的法律法规以及国 家出台的相关网络安全的法律条文, 形成本校独特的 网络安全法律体系, 同时可以设立网络安全维护小组, 实时监督新媒体的使用。

\section{2. 增强信息安全管理}

针对网络安全问题实时监控, 提倡早发现, 早维 护, 确保问题能够得到及时解决, 减少不必要的网络 安全风险。同时可以定期设立网络安全测试, 对于信 息安全问题的处理设立严格的奖惩机制。还应该建设 相关网络安全维护团队, 团队中应该有在建设, 维护 等不同领域的专业人员, 并且针对他们的薄弱环节进 行定期培训, 在全方面提升他们的专业业技能。除了 以上外部因素, 最重要的是新媒体使用内部因素的维 护, 在各种网络设备中, 用电安排, 权限设置, 和系 统配备等方面加强监督, 在信息泄漏不良信息传播的 方面, 应该加强防火墙的功能, 以保证网络使用过程 中的安全性, 同时在储存信息方面, 可以策划多种途 径的储存方案, 例如云储存等来保证信息的完整性。

\section{3 可以将 $C I O$ 体制引入到新媒体使用当中}

首席信息执行官, 其英文简称 CIO (Chief Information Officer)。其主要的功能是负责参与学 校的信息战略规划。十三五规划当中，教育科技司司 长则表示高校在进行新媒体使用的过程中, 应当引入 首席信息执行官制度。把这一项制度引入到学校的信 息管理中, 有利于学校信息管理的然后运行也有利于 在技术层面上实现有效的沟通, 使得学校的信息安全 得到进一步的保障。

\section{4 提升新媒体专业人士的素养}

新媒体不同于传统媒体, 具有个体特性、广泛的 社会性以及主体性等特征, 使得学习者必须要具有一 定的使用能力和判断能力。为提高新媒体在高校行政 管理当中的应用, 也应当提高相关的新媒体人才的专 业素养。使得这些新媒体专业人才能够更加友好的进 行新媒体创造管理和互动交往。首先, 在高校行政管 理当中正确的使用新媒体, 就需要培养相关师生的新
媒体使用意识，使得相关的使用者能通过相关理论的 学习与一定实践的操作, 迅速的了解到新媒体的使用 方法与操作手段, 使得新媒体能够更好的服务于高校 的行政管理。其次, 相关的自媒体专业人才需要拥有 终身学习的能力, 这种能力要求相关的使用人员要不 断的巩固自己已经学习的知识, 并且在这种知识的基 础上努力的吸收新的知识, 用新的知识与之前所学的 知识进行融合, 然后能够更好的应用于工作和实践当 中去。

\section{5 加强对信息化人才的培养}

进行信息化人才的培养, 就要求高校制定相关的 信息化人才培养的方案，包括培养体系以及培养的具 体内容的。可以从高校的信息化专业角度出发, 与相 关的 it 企业以及相关的 it 行业, 或者从事软件开发 和软件测试等企业或者行业建立一定的人才输送政 策, 最好可以形成产学研研究基地, 通过产学研研究 的方式进行一定的人才打造和人才培养。同时, 在进 行相关人员培养的过程中, 除了应当重视相关人员专 业知识上面的培养, 还应当重视相关人员人格上面的 塑造, 包括三观的塑造等, 此外, 还应当重视相关人 员的个性化发展。

除了通过学校和企业合作以外, 还可以使得学校 自己建立一支具有研发技术的教师队伍, 使得该教师 队伍参与对外的相关新媒体信息化方面的研讨。通过 这类研讨会的形式, 将本校的新媒体信息化队伍所学 到的知识, 与外校的新媒体信息化队伍所接触的知识, 进行不断的融合和发展, 从而形成一个更加严密、更 加具有逻辑性和更加能够实践的新媒体信息化专业 知识体系。

其次, 还可以建立相关的研究基金, 这个研究基 金可以是通过国家财政或者当地政府的财政拨款，也 可以是通过企业的拨款, 或者高校自建的基金池内的 拨款获得的。这一部分基金运用于腺载体信息化项目 的研究, 可以给予相应的研究人员一定的资金支持和 资金奖励。

最后, 除了可以建立校企合作以及相关的人员培 养基金以外，还可以通过与政府合作。当然这一切都 是建立在拥有一个合理的法律法规的保障下, 以及相 关的激励机制的作用下。因此，在进行信息化人才的 培养的过程中, 应当注重相关法律法规的完善和相关 激励机制的建立, 通过这些完善的法规和相关的激励, 吸引相关的人才留下来发展。

\section{6 借鉴外国的新媒体在高校行政管理当中 运用的经验}

由于国外的新媒体在高校行政管理当中运用的 时间较早, 因此, 已经形成了一个完整的体系。我国 的新媒体技术运用在高校的行政管理当中, 就可以借 鉴欧美等发达国家的经验。这种借鉴并不是全盘地照 
抄，而是经过一定的实地考察、观察美国以及欧洲等 发达国家, 如何将新媒体良好的运用到高校行政管理 当中的技术手段, 并且进行一定的总结。在进行了相 关的学习与总结之后, 再将学习到的精华运用于我国 当中。在运用的过程当中也可以采用先试点的方式, 试点的效果好, 再进行大规模的推广, 而不是一味的 想当然的直接进行大规模的推广。

\section{5. 结论:}

在信息化互联网迅速发展的当下，作为信息最时 效化传播的媒体也经历了翻天覆地的变化, 各大新媒 体竞相出现, 新兴媒体正成为当今热潮。新媒体贯穿 在我们生活中, 亦然也成了我们当今校园的一部分。 我们的教学学习方法及作息生活因新媒体技术的到 来也有了相应的改变, 更加方便便捷, 极其具有个性 化的充实我们的生活。根据数据显示, 目前我国已至 少有 8.02 亿网民, 互联网的普及率达到 57.7\%, 国内 4G 网络覆盖率达到 99\%。显然, 在新媒体时代, 院校 必须经过互联网十及信息化教育来达到培养优秀信 息专业人才目标, 不断引进新理念教育模式, 创新开 放教育体系, 从而推进新媒体时代 “互联网+” 的技 术及相关理念改革。

\section{REFERENCES}

[1] Fan Xinai. Research on the current situation, problems and Countermeasures of new media in Colleges and universities [J]. Journal of Henan University of Technology: Social Science Edition, 2018 (2): 78-82

[2] Xing Yunfei, Wang Xiwei, Han Xuewen, et al. Research on the influence of network nodes under the new media environment based on information entropy -- Taking WeChat official account as an example [J]. library and information work, 2018 (5):76-86.

[3] Liu Hui. Research on the influence and application of new media on University Administration [J]. New media research, 2016, (10), 49-49, 51

[4] Xu Yunlu, Zhang Yifeng, Yang Tian Yi. The development status and future trend of WeChat official account on campus. [J]. news communication, 2016 (6): 31-32, 34.

[5] Cai Qingxiang. Problems and Countermeasures of organizational communication of university administrative organizations under the network environment [J]. China urban economy, 2011, (24). 274-275

[6] Wen Ya, Cui Lijun. Exploration of student management in medical colleges and universities [J]. China Medical Guide, 2014, (11). 149-151155
[7] Li Silin. Analysis of the current situation, problems and improvement measures of China's University Administration [J]. China market, 2015, (43). 133134

[8] Chen Hao. Professional construction of administrative personnel in Colleges and Universities -- Based on the perspective of the conflict between academic power and administrative power [J]. Shandong higher education, 2016, (1). 13-18

[9] Duan Mengjiao. Analysis on the current situation and Innovation Countermeasures of administrative management in private colleges and universities [J]. Theoretical research and practice of innovation and entrepreneurship, 2019, (6). 81-82

[10] Han Qing. Research on incentive mechanism of administrative personnel in private colleges and Universities -- Taking Wuhan Bioengineering University as an example [D]. Central China Normal University, January 44, 2015 Vocational and technical college, 2019, (6). 71-72 